\title{
SANGRADO GASTROINTESTINAL EN PACIENTES HOSPITALIZADOS
}

\author{
Juan José Diaztagle F.MD*, Roberto H. Cruz B. **, David F. Eslava O**, Juan F. Briceño P.**, Andrés C.
} Hernández A**, Natalia Jiménez M.**, Mónica Jiménez M.**, Laura C. Melo F.**, Ronald E. Sierra M.**, Leonidas Vásquez H MD***

\section{Resumen}

El sangrado gastrointestinal es una complicación potencial del paciente que se hospitaliza. Como las descripciones iniciales en cuidados intensivos relacionaban el sangrado por úlceras de estrés con alta mortalidad, la investigación fue creciente en los pacientes críticos. No sucedió lo mismo con los hospitalizados y los trabajos disponibles son escasos. Los principales factores de riesgo se han descrito en los críticos (ventilación mecánica más de 48 horas y coagulopatía). La eficacia de la terapia no se ha demostrado en aquellos en la sala general. La evidencia indirecta muestra utilidad marginal de estos medicamentos en la prevención del sangrado gastrointestinal. En los casos críticos hay abundante información pero la interpretación es confusa. Se ha documentado una tasa alta de formulación para prevenir el sangrado en los hospitalizados, la mayoría sin indicación clara, lo cual puede exponer al paciente a eventos adversos innecesarios y gastos adicionales al sistema de salud. Se recomiendan estrategias para implementación de guías para el uso racional de estos fármacos.

Palabras clave: úlceras de estrés, profilaxis, sangrado gastrointestinal, terapia de supresión ácida, inhibidores de bomba de protones, antagonistas de receptores de histamina tipo 2.

Abreviaturas: SGI, sangrado gastrointestinal; SRMD, stress-related mucosal disease; AINES, antiinflamatorios no esteroideos; ASHP, American Society of Health-System Pharmacists; FDA, Food and Drug Administration; ERGE, enfermedad por reflujo gastroesofágico.

\section{GASTROINTESTINAL BLEEDING IN HOSPITALIZED PATIENTS}

\section{Abstract}

Gastrointestinal bleeding is a potential inpatient complication. Research has extensively focused in critically ill patients due to initial descriptions of high mortality related to bleeding due to stress ulceration. On the other hand, research evaluating GI bleeding in inpatients is scarce with few available reports. The main risk factors have been described in critically ill patients (requiring mechanical ventilation for more than 48 hours and coagulopathy). Treatment efficacy has not been demonstrated in patients hospitalized in the general ward. Indirect evidence show

Fecha recibido: diciembre 21 de 2012 - Fecha aceptado: agosto 14 de 2013

* Especialista en Medicina Interna, Epidemiólogo. Magíster en Fisiología. Instructor Asistente, Fundación Universitaria de Ciencias de la Salud. Hospital de San José. Profesor Asociado, Departamento de Ciencias Fisiológicas, Facultad de Medicina, Universidad Nacional. Bogotá DC, Colombia.
** Estudiantes de la Facultad de Medicina, Fundación Universitaria de Ciencias de la Salud. Bogotá DC, Colombia.

*** Residente III de Medicina Interna, Fundación Universitaria de Ciencias de la Salud. Bogotá DC, Colombia. 
marginal usefulness of these medications in gastrointestinal bleeding prevention. There is plentiful data on critically ill patients but with confusing interpretation. It has been documented that preventive medicines are often prescribed to avoid bleeding in inpatients, with no clear indication in most cases, exposing the patient to unnecessary adverse events and additional costs for the healthcare system. Implementation guidelines are recommended for the rational use of these medicines.

Key words: stress ulceration, prophylaxis, gastrointestinal bleeding, acid suppression therapy, proton bomb inhibitors, histamine type 2 receptor antagonists

\section{Introducción}

El sangrado gastrointestinal (SGI) es una complicación potencial del paciente hospitalizado. En 1969 Skillman y col. reportaron un síndrome clínico de "ulceración por estrés", el cual resultó fatal en siete de 150 hospitalizados en forma consecutiva en cuidados intensivos, los cuales tenían en común falla respiratoria, hipotensión y sepsis. El examen posmortem mostró múltiples úlceras superficiales localizadas en especial en el fondo del estómago. Posterior a estas descripciones, la utilización de la profilaxis para el SGI en pacientes críticos fue una conducta común y se realizaron múltiples estudios para evaluar su eficacia y factores de riesgo en este contexto. ${ }^{1}$

Esta condición patológica ha recibido varios nombres entre los cuales las "úlceras de estrés" y la "enfermedad de la mucosa relacionada con el estrés (SRMD)" son dos de los que se utilizan con mayor frecuencia. La SRMD se ha definido como una lesión inflamatoria aguda de la mucosa gástrica que se produce cuando un individuo es sometido a demandas físiológicas elevadas. ${ }^{2}$ En términos generales, las úlceras por estrés son defectos en la mucosa gastroduodenal que pueden ser únicas o múltiples y causan un espectro de manifestaciones clínicas que van desde erosiones de la mucosa superficial, pasando por ulceración, hasta hemorragia que puede ser mortal.

El riesgo es mayor en la unidad de cuidados intensivos (UCI), donde se ha encontrado evidencia endoscópica de SRMD entre 75 y $100 \%$ en las primeras 24 horas del ingreso. Estas úlceras representan un peligro de hemorragia, que se puede asociar con anemia, inestabilidad hemodinámica y necesidad de transfusión en cerca de 1 a $4 \%$ de los pacientes en estado crítico, con una tasa de mortalidad que se acerca al 50\%. ${ }^{3}$ Por esta razón, la administración de fármacos para prevenir el SGI o terapia de supresión ácida se realiza con bastante frecuencia.

La situación en el que se hospitaliza en la sala general no está bien definida. En este contexto, la utilización de estos medicamentos está sujeta a discusión y el beneficio clínico no es muy claro. En el presente artículo se hará una breve revisión acerca de los factores de riesgo de SGI, utilización de los medicamentos de supresión ácida, posibles eventos adversos y recomendaciones generales para su uso, en especial en pacientes no críticos.

\section{Fisiopatología, incidencia y factores de riesgo en hospitalizados}

La SRMD ocurre debido a interacciones complejas de factores que producen lesión en el lumen gástrico, reducen el flujo sanguíneo y alteran el pH intramucoso, de tal forma que dañan los mecanismos de defensa de la mucosa gástrica. Si bien la acidez aparece como esencial para la generación de úlcera por estrés, no es el único factor patogénico. Por otra parte, la isquemia de la mucosa es un factor decisivo ya que disminuye la capacidad para neutralizar el hidrógeno y contribuye a la acidosis intramural, la muerte celular y la ulceración. La isquemia también puede comprometer el 
metabolismo energético y dañar los procesos reparativos. Otros factores de injuria intraluminal que incluyen la bilis y la pepsina no tienen establecido por completo su papel. ${ }^{4}$ Puesto que el ácido parece estar implicado en la patogénesis de estas lesiones, se espera que los tratamientos supresores de este tengan el potencial de prevenir la aparición de SRMD.

La incidencia del SGI en pacientes hospitalizados es variable. Estudios realizados en UCI han reportado una significativa de SGI que varía entre 0,6 y $6 \%$, que son tasas más bajas que las informadas antes., ${ }^{5,6}$ En el contexto de la hospitalización general la incidencia también es baja. Un estudio realizado entre 535 pacientes hospitalizados en una sala de medicina interna mostró que se presentó SGI con significación clínica en un solo paciente $(0,2 \%){ }^{7}$ Otro estudio realizado en la Cleveland Clinic evaluó durante un período de cuatro años 17.707 hospitalizados en la sala de medicina interna general, de los cuales sangraron $73(0,41 \%) .{ }^{8}$ Mientras que una investigación llevada a cabo en un hospital en Boston durante cuatro años, evaluó una cohorte de 74.394 admisiones, entre los cuales 224 pacientes presentaron SGI $(0,29 \%){ }^{9}$

La variación en la incidencia puede explicarse por dos aspectos fundamentales. En primer lugar, las características de la población que se estudia, teniendo en cuenta que el SGI varía según la prevalencia de los factores de riesgo, la formulación o no de profilaxis farmacológica para el sangrado y el tipo de nutrición que recibe, en especial el paciente crítico en quien la enteral puede ser un factor de protección para el sangrado. En segundo lugar, la definición de SGI que se ha utilizado para identificar un paciente con esta condición ha variado en los estudios. Un término que se ha venido utilizando con mayor frecuencia es el de SGI "clínicamente significativo", el cual hace referencia a un sangrado aparente asociado con inestabilidad hemodinámica o disminución de la hemoglobina que requiere transfusión. Es evidente que la incidencia cambia si se tiene en cuenta cualquier sangrado a uno con significación clínica.

En cuanto a los factores de riesgo para SGI, gran parte de los estudios que los han evaluado se han realizado en el contexto del cuidado crítico. Uno de los más representativos publicado por Cook y col. en $1994^{10}$, muestra que la falla respiratoria ameritó ventilación mecánica por más de 48 horas y la coagulopatía (definida como recuento de plaquetas $<50.000 / \mathrm{ml}$, tiempo de protrombina 1,5 veces mayor que el control o tiempo de tromboplastina tisular dos veces más que el control) fueron factores de riesgo independientes y fuertes para el sangrado clínico importante (OR de 15,6 y 4,3 en forma respectiva). Los pacientes que tuvieron uno o ambos factores, desarrollaron sangrado clínico importante en 3,7\% (IC 95\%: 2,5-5,2) de los casos, comparado con $0,1 \%$ (IC 95\%: 0,02-0,5) entre los que no los presentaban.

En otras publicaciones, se han identificado más factores de riesgo para SGI. Entre ellos se puede mencionar insuficiencia renal aguda, sepsis, hipotensión, trauma mayor, quemaduras, cirugía mayor, trauma craneoencefálico, historia de SGI, falla orgánica múltiple, dosis altas de corticoides, ingesta de anticoagulantes y enfermedad hepática. En estos reportes la posibilidad de sangrado se elevaba con el aumento del número de factores de riesgo. ${ }^{11-14}$

Estos factores se pueden presentar en el contexto del paciente hospitalizado en sala general. De hecho algunos en los pisos provienen de la UCI, en donde pudieron estar expuestos a ellos. El tiempo que dura el riesgo de SGI cuando cursa con una de estas condiciones es incierto y variable, sobre todo con aquellos factores que son transitorios. Por otra parte, en los casos críticos la interacción de muchos de los factores con el estado de la nutrición recibida es clave a la hora de analizar el riesgo de SGI. Por lo tanto, si bien es cierto que se debe tener en cuenta la evidencia científica descrita en la UCI, no es fácil extrapolarla al hospitalizado y tal vez sea uno de los motivos por los cuales en algunas investigaciones en medicina interna excluyen el paciente que estuvo antes en cuidados intensivos.

En cuanto a los hospitalizados en sala general hay pocas evaluaciones. ${ }^{8.15}$ Terdinam y col. publicaron un estudio de casos y controles que tuvo como objetivo determinar los factores de riesgo, la causa y los resul- 
tados del SGI en pacientes hospitalizados. ${ }^{15}$ Durante el período de estudio 13.330 fueron hospitalizados, de los cuales 67 cumplieron con la definición de caso. Los factores de riesgo asociados con SGI fueron: estancia en UCI, ventilación mecánica, uso de vasopresores, sepsis, enfermedad hepática, coagulopatía o INR elevado. Sin embargo, en el análisis multivariado solo la estancia en UCI (OR=2,5; IC 95\%, 1,0-6,1) y la ventilación mecánica $(\mathrm{OR}=3,4$; IC 95\%, 1,0-11) permanecieron como factores independientes. Cuando se analizaron 24 casos que no estuvieron en UCI sobre la relación de nutrición parenteral, antecedente de trasplante de órgano y enfermedades malignas con SGI, solo la cirugía mayor como asociación negativa tuvo significancia estadística en el análisis multivariado $(\mathrm{OR}=0,15$; IC 95\%, 0,04-0,6).

Por su parte, Qadeer y col. ${ }^{8}$ realizaron un estudio retrospectivo de casos y controles con el objetivo de identificar factores de riesgo que podrían predecir SGI intrahospitalario y evaluar si la profilaxis con terapia de supresión ácida tuvo menor tasa de sangrado. Entre 17.707 enfermos que ingresaron a hospitalización en una sala de medicina interna general, 73 presentaron SGI. El principal factor de riesgo identificado en este estudio fue el tratamiento con dosis plenas de anticoagulantes o clopidogrel (OR=5,4; IC 95\%, 2,6-11,7). Vale la pena mencionar que el uso de antiinflamatorios no esteroideos (AINES), aspirina o corticoides no fueron factores de riesgo significativos. Los autores concluyeron que el SGI es infrecuente en pacientes no críticos y que la anticoagulación aparece como el factor de riesgo más importante.

\section{Eficacia de la profilaxis contra la úlcera de estrés en la sala general}

Pocos estudios evalúan la eficacia de la profilaxis de la úlcera de estrés en el paciente hospitalizado en medicina general. Estruch y col. ${ }^{16}$ realizaron uno aleatorio controlado con placebo, para valorar la eficacia de magaldrate en la prevención del SGI agudo en hospitalizados con enfermedades graves en un hospital universitario de España. Incluyeron aquellos con insuficiencia respiratoria o cardíaca, sepsis, enferme- dad cerebrovascular y falla hepática o renal, o bien que recibían tratamiento con corticoides, heparina o warfarina. En los resultados, el magaldrate redujo la tasa de SGI de manera significativa $(\mathrm{p}<0,001)$. Grau y col. ${ }^{17}$ efectuaron un estudio aleatorio controlado donde compararon la eficacia de cimetidina y sucralfate en la prevención del SGI en población de medicina general, utilizando los mismos criterios de inclusión descritos en el estudio anterior. El sangrado fue semejante en los dos grupos, pero no hubo un control con placebo.

Estos estudios tienen limitaciones metodológicas que no permiten extrapolar los resultados. En primer lugar, la mayoría tenían enfermedades críticas, con un gran porcentaje de falla ventilatoria y múltiples factores de riesgo para SGI, como ingesta de corticoides en dosis altas y warfarina. En segundo lugar, no hubo una distinción entre sangrados mayores y menores, lo cual es importante en estos casos. Además el magaldatre no está disponible en el comercio. Por lo anterior las guías publicadas por la American Society of Health-System Pharmacists (ASHP) no percibieron que estos datos fueran adecuados para realizar una recomendación rutinaria de la profilaxis contra la úlcera de estrés. ${ }^{18}$

La evidencia indirecta también demuestra que en pacientes no críticos hospitalizados, la terapia de supresión ácida parece tener eficacia limitada para prevenir la hemorragia digestiva alta. Herzig y col. evaluaron una cohorte de 78.394 adultos (edad media 56 años, $41 \%$ varones) que ingresaron al hospital durante tres días o más con diagnósticos diferentes a hemorragia digestiva alta. De estos, 45.882 (59\%) tenían medicamentos antiácidos, inhibidores de la bomba de protones o antagonistas de los receptores de histamina tipo 2; $224(0,29 \%)$ presentaron hemorragia digestiva alta nosocomial. Al realizar un análisis de propensión, la terapia de supresión ácida se asoció con una menor incidencia de hemorragia digestiva alta nosocomial (OR=0,63; IC 95\%, 0,42-0,93). Aunque hubo un ligero beneficio, el NNT fue de 770, lo cual es necesario tener en cuenta a la hora de tomar decisiones. ${ }^{9}$ Otros estudios han mostrado resultados diferentes, tal es el caso de la investigación ya mencionada de Qadeer y col. en la cual la administración de profilaxis gástrica iniciada de novo o en pacientes 
que ya venían recibiéndola antes de la hospitalización, no fue factor preventivo del sangrado $(\mathrm{OR}=1,4$; IC $95 \%, 0,7-3,5){ }^{8}$

En pacientes críticos la situación es diferente, pues se han realizado varios estudios aleatorios para evaluar la eficacia de estos medicamentos. ${ }^{19,20}$ Sin embargo, el análisis de esta información ha sido compleja dado que los resultados y los diversos metanálisis han sido discordantes ${ }^{21-23}$, incluyendo algunos de publicación reciente. ${ }^{1,24}$ Esta situación ha sido favorecida además porque el tema no solo es de eficacia de estos fármacos, sino también de seguridad en su administración, teniendo en cuenta la hipótesis del aumento del riesgo de neumonía asociada con el ventilador en quienes reciben estas terapias. De tal forma que una situación clara y definida en cuanto al beneficio y seguridad no está bien establecida para el paciente crítico.

\section{Uso inadecuado de la terapia de supresiónácida en hospitalizados}

Los fármacos para supresión ácida son utilizados con alta frecuencia en pacientes hospitalizados. Los estudios que han evaluado su uso estiman que entre el 40-70\% de los pacientes no quirúrgicos reciben alguno para supresión ácida. ${ }^{9}$ En ocasiones se inicia "de novo" durante la estancia, mientras otros ya lo venían recibiendo. Este alto porcentaje de utilización al parecer no es adecuado en la mayoría de los casos, según el reporte de casi todos los estudios que han evaluado este tema. ${ }^{25-32}$

Nardino y col. evaluaron en forma prospectiva el uso de la terapia de supresión ácida en 122 de 226 (54\%) en pacientes hospitalizados en medicina general durante un período de tres meses. De ellos, $35 \%$ tuvieron indicación adecuada mientras que $65 \%$ no. ${ }^{21}$ Por su parte, Noguerado y col. revisaron en forma retrospectiva 209 historias clínicas de hospitalizados en medicina interna para evaluar el uso adecuado de la terapia de supresión ácida. Definieron "uso" y "utilización adecuada" de la misma manera que en el estudio previo de Nardino y col. En los resultados se encontró que de $75,1 \%$ que la recibieron el 72,2\% no tenía indicación adecuada. ${ }^{27}$
Zink y col. en un estudio retrospectivo en hospitalización general, caracterizaron la población que egresaba con algún medicamento para la supresión ácida. Se evaluaron 324 casos de los cuales 128 (40\%) recibieron terapia de supresión ácida de forma adecuada y 196 (60\%) inadecuada. El 34\% de este último grupo recibió alguno de estos medicamentos al egreso y un alto porcentaje continuó en forma impropia durante tres a seis meses de seguimiento. ${ }^{28}$

Hay trabajos que muestran hallazgos semejantes en otros países. Khudair y col. lo hicieron durante dos meses en hospitalizados en un hospital universitario de Qatar. De los 389 pacientes recolectados, la terapia de supresión ácida fue ordenada al 53\%, de los cuales $34 \%$ tuvieron alguna indicación, mientras que $66 \%$ no. ${ }^{29}$ En Líbano, una investigación mostró que entre 1.004 pacientes de la sala general, $71,6 \%$ recibieron esta terapia. Cuando se comparó con las guías de la ASHP, el uso fue inadecuado en $87,6 \% .^{30} \mathrm{La}$ investigación en Suiza en pacientes quirúrgicos de Bez y col. demostró que la profilaxis fue dada a $79 \%$ que no tenía ningún factor de riesgo para SGI. ${ }^{26}$ Un estudio retrospectivo realizado en un hospital de la Florida (Estados Unidos) mostró también utilización inapropiada de terapia de supresión ácida en $73 \%$ de los casos. ${ }^{32}$

Hay dos aspectos fundamentales cuando se analizan estos estudios. El primero es definir qué significa "uso" o "utilización" del fármaco. Nardino y col. consideran "uso", como cualquier prescripción para terapia de supresión ácida independiente de la dosis, siempre y cuando el paciente haya recibido por lo menos una dosis. ${ }^{26}$ Lo que propone este autor es una definición operativa que estandarice la recolección del dato, la cual es necesaria en estos casos. Sin embargo, no hay que perder de vista que el análisis de esta variable no es tan simple, ya que es evidente que no es lo mismo la prescripción del fármaco un solo día de la hospitalización, que hacerlo durante todo o la mayor parte del tiempo.

El segundo punto y tal vez el más relevante es la definición de prescripción o uso "adecuados". No existe un consenso amplio a partir del cual definir cuál paciente debe recibir o no la profilaxis. De hecho, la guía más reconocida y citada es la de la 
ASHP, la cual fue publicada en 1999 y en la que se basan con frecuencia los investigadores. Muchos autores también utilizan las indicaciones aprobadas por la FDA o las que aparecen en la literatura médica. En este punto se sobrepone el concepto de "profiláctico" y "terapéutico", ya que las indicaciones de la FDA son para el tratamiento de ciertas patologías en las cuales se formula este medicamento, mientras que en hospitalizados se fundamenta en un concepto de profilaxis, es decir prevenir un evento en un grupo de pacientes que está expuesto a un riesgo de padecerlo.

Para Nardino y col. ${ }^{26}$ las indicaciones adecuadas se fundamentaron en las aprobadas por la FDA y otras que consideraron bien soportadas en la literatura médica. Estas incluyeron: enfermedad ulcerosa activa, mantenimiento en pacientes con úlcera de alto riesgo, esofagitis erosiva o gastritis, reflujo gastroesofágico sintomático, profilaxis en pacientes de alto riesgo (coagulopatía o ventilación mecánica prolongada) y tratamiento contra $H$. pylori. También se consideraron como indicaciones aceptables el alivio de la dispepsia asociada con AINES, prevención de úlceras por este fármaco y pruebas terapéuticas para dispepsia no ulcerosa. Zink y col. ${ }^{28}$ utilizaron las indicaciones descritas en el empaque: ERGE, infección por H.pylori, esofagitis, terapia de mantenimiento para úlceras duodenales activas, esofagitis por reflujo y tratamiento a largo plazo de enfermedad hipersecretora, úlcera duodenal o gástrica. Ellos adicionaron como indicaciones aceptadas: gastritis, alivio de la dispepsia y prevención de las ulceras inducidas por AINES en pacientes con alto riesgo. Por su parte, para Kudhair y col. ${ }^{29}$ la terapia fue justificada si se basaba en las recomendaciones realizadas por la ASHP, la información de prescripción aprobada por la FDA y cierta evidencia fuerte de la literatura. Las recomendaciones incluyeron úlceras gástricas o duodenales benignas, ERGE sintomática, esofagitis erosiva, profilaxis de aspiración ácida, tratamiento de $H$. pylori, condiciones hipersecretoras patológicas, prevención de úlceras por toma de AINES (en un grupo de pacientes específicos), profilaxis de úlceras de estrés en pacientes con alto riesgo, cirrosis hepática, trasplantados y los que reciben corticoides más AINES.
Por último se han explorado las causas de utilización inadecuada de estos fármacos. Hussain y col. ${ }^{33}$ realizaron un estudio de corte transversal basado en una encuesta virtual que evaluó los conocimientos, creencias y comportamientos de los médicos al realizar la profilaxis para SGI con el objetivo de evaluar las causas de la prescripción inadecuada. En sus resultados, los factores asociados con mayor prescripción fueron el temor al SGI, posibles repercusiones legales por no hacerlo y el desconocimiento de eventos adversos de los medicamentos utilizados para la terapia de supresión ácida. También se han documentado las variables que pueden intervenir como el incremento en la edad, el género masculino, la duración de la estancia hospitalaria y la presencia de comorbilidades. ${ }^{34}$

\section{Complicaciones a largo plazo e impacto económico de una terapia inadecuada de supresión ácida}

Hay dos aspectos importantes a la hora de evaluar el uso innecesario de este tratamiento en hospitalizados: el riesgo potencial de efectos adversos y la generación de gastos innecesarios para el sistema salud.

Efectos adversos: se mencionan la enfermedad por Clostridium difficile (CDAD), neumonía y complicaciones óseas como osteoporosis o aumento del riesgo de fracturas. Dial y col..$^{35}$ encontraron en un estudio realizado en el Reino Unido, riesgo aumentado para CDAC con RR de 2,9 (IC 95\% 2,4-3,4) para los inhibidores de la bomba de protones y 2,0 (IC 95\% 1,6-2,7) para antagonistas $\mathrm{H}_{2}$. Mientras que otro en Canadá reveló riesgo aumentado de CDAC relacionado solo con el uso de inhibidores de la bomba de protones $\left(\mathrm{OR}=2,7\right.$; IC 95\%1,4-5,2). ${ }^{36}$ Vale la pena anotar que no todas las evaluaciones han demostrado esta asociación. ${ }^{37}$

En cuanto a la neumonía, se ha encontrado que el uso de inhibidores de bomba de protones o antagonistas $\mathrm{H}_{2}$ aumenta la frecuencia casi al doble. ${ }^{38}$ Herzig y col. hallaron asociación entre terapia de supresión ácida y neumonía adquirida en el hospital en 63.878 admisiones, con significación estadística en cuanto a 
inhibidores de bomba de protones $(\mathrm{OR}=1,3$; IC $95 \%$ $1,1-1,4)$, mientras que para inhibidores $\mathrm{H}_{2}$ no $(\mathrm{OR}=1,2$; IC $95 \% 0,98-1,4){ }^{39}$

Por otra parte, estos medicamentos pueden aumentar el riesgo de fracturas. Una revisión realizada por Laine L, lo evidenció en tres estudios con el uso de inhibidores de bomba de protones, aunque la fuerza de la asociación no fue muy grande. ${ }^{40}$ Hace poco un metanálisis demostró esta asociación ( $\mathrm{OR}=129$; IC 95\% 1,18-1,41), mientras que con el uso de antagonistas $\mathrm{H}_{2}$ no la hubo (OR=1,1; IC 95\% 0,99-1,23). ${ }^{41}$

Costos: Erstad B. y col. ${ }^{42}$ los analizaron en dos fases, en la primera se realizó medición basal de estos y en la segunda se calcularon los mismos costos pero después de una intervención educativa hospitalaria que tuvo como objetivo disminuir el uso inapropiado. Las cifras fueron de USD\$2.272 en la primera fase y USD\$ 1.417 en la posterior. Wadibia E. y col. ${ }^{43}$ por su parte examinaron el costo de la terapia inapropiada para la profilaxis de la úlcera de estrés en pacientes admitidos a una UCI y fueron calculados en USD\$ 5.084. Por último, Heidelbaugh e Inadomi ${ }^{44}$ analizaron los efectos económicos de la profilaxis inapropiada en los servicios de medicina familiar y medicina interna general observando que ascendió a USD\$ 11.000 en cuatro meses.

\section{Recomendaciones actuales sobre profilaxis del SGI en el hospitalizado}

Hay unas claras en cuanto a la UCI en razón a la mayor información científica, a diferencia de la hospitalización general, teniendo en cuenta que los factores de riesgo y la eficacia se han documentado en forma más consistente. ${ }^{45,46}$ Existen pocas sociedades científicas en el mundo que ofrecen recomendaciones basadas en la evidencia. Tal vez la guía más reciente para la profilaxis de la úlcera de estrés fue publicada por la ASHP en 1999, donde no se autoriza para el paciente médico o quirúrgico que no se encuentre hospitalizado en UCI. ${ }^{18}$

Un campo en el cual existen recomendaciones generales en cuanto a la prevención de SGI es en el de los pacientes que reciben AINES. Al respecto The American College of Gastroenterology publicó sus guías en las cuales las medidas preventivas dependen del riesgo de complicaciones relacionadas con la ingesta crónica de AINES. El paciente se clasifica en tres categorías de riesgo: alto para aquellos con historia de úlceras complicadas o con más de dos factores de riesgo; medio, cuando concurren uno o dos de los siguientes factores: edad > 65 años, dosis altas de AINES, historia de úlcera no complicada y uso concomitante de aspirina, corticoides o anticoagulantes; bajo, cuando no hay ninguno de los factores de riesgo mencionados. De tal forma que en este último no está indicada la terapia de supresión ácida, en los de riesgo medio se aconseja profilaxis con misoprostol o inhibidores de bomba de protones y en los de alto está indicada también la terapia de supresión ácida con misoprostol o inhibidores de bomba de protones y además se sugiere una alternativa a AINES, por ejemplo, un inhibidor de la ciclooxigenasa 2 (COX2). ${ }^{47}$

Se destaca el documento Practice management guidelines for stress ulcer prophylaxis actualizado en 2008 que da recomendaciones en cuanto al SGI en el paciente crítico $^{48}$ (Tabla 1). Otro documento importante es Management of acute upper gastrointestinal bleeding: NICE guidance ${ }^{49}$ En este se recomienda dar terapia de supresión ácida (antagonistas de los receptores H2 o IBP) para la prevención primaria de la hemorragia gastrointestinal alta en pacientes graves ingresados en UCI. Si es posible, utilizar la forma oral del medicamento, basado en evidencia de muy baja calidad de estudios controlados aleatorios y en datos económicos de aplicación parcial y con serias limitaciones.

Por último, aunque no está explícito en las guías de manejo, se ha demostrado que la implementación de diversas medidas para disminuir el uso inadecuado de terapia de supresión ácida puede ser benéfica. En un estudio de antes y después se demostró que el uso de una guía estandarizada al ingreso del paciente al hospital, redujo el inicio impropio de inhibidores de bomba de protones en un $10 \%(\mathrm{p}=0,001){ }^{50}$ También se han demostrado disminuciones significativas en el uso inadecuado después de intervenciones de farmacia, para la aplicación de guías en hospitalización. ${ }^{51,52}$ Por 


\section{Tabla I. Profilaxis del sangrado gastrointestinal en el paciente crítico}

\begin{tabular}{|c|c|c|c|}
\hline & Recomendación Clase I & Recomendación Clase II & Recomendación Clase III \\
\hline $\begin{array}{l}\text { ¿Cuáles son los facto- } \\
\text { res de riesgo para el } \\
\text { desarrollo de úlcera de } \\
\text { estrés y qué pacientes } \\
\text { requieren profilaxis? }\end{array}$ & $\begin{array}{l}\text { Se recomienda la profi- } \\
\text { laxis para todos los pa- } \\
\text { cientes con: } \\
\text { I. Ventilación mecánica } \\
\text { 2. Coagulopatía } \\
\text { 3. Lesión cerebral } \\
\text { traumática } \\
\text { 4. Lesiones graves por } \\
\text { quemadura }\end{array}$ & $\begin{array}{l}\text { Se recomienda la profi- } \\
\text { laxis para todos los pa- } \\
\text { cientes de UCl con: } \\
\text { I. Politrauma } \\
\text { 2. Sepsis } \\
\text { 3. Insuficiencia renal } \\
\quad \text { aguda }\end{array}$ & $\begin{array}{l}\text { Se recomienda la profilaxis para todos } \\
\text { los pacientes de UCl con: } \\
\text { I. ISS >I5 (Injury Severity Score) } \\
\text { 2. Tratamiento con esteroides a dosis } \\
\text { altas (> } 250 \mathrm{mg} \text { hidrocortisona o equi- } \\
\text { valente por día) } \\
\text { 3. En pacientes seleccionados no es ne- } \\
\text { cesaria la profilaxis }\end{array}$ \\
\hline $\begin{array}{l}\text { ¿Existe un agente pre- } \\
\text { ferido para la profilaxis } \\
\text { de la úlcera de estrés? } \\
\text { Si es así, ¿cuál es? }\end{array}$ & $\begin{array}{l}\text { No hay diferencia en- } \\
\text { tre los antagonistas } \mathrm{H} 2 \text {, } \\
\text { agentes citoprotectores y } \\
\text { algunos inhibidores de la } \\
\text { bomba de protones. } \\
\text { Los antiácidos no deben } \\
\text { utilizarse como profilaxis } \\
\text { de úlcera de estrés }\end{array}$ & $\begin{array}{l}\text { Los compuestos que con- } \\
\text { tienen aluminio no deben } \\
\text { utilizarse en pacientes en } \\
\text { diálisis }\end{array}$ & $\begin{array}{l}\text { La alimentación enteral por sí sola } \\
\text { puede ser insuficiente para la profilaxis } \\
\text { de úlcera de estrés }\end{array}$ \\
\hline $\begin{array}{l}\text { ¿Cuál es la duración de } \\
\text { la profilaxis? }\end{array}$ & $\begin{array}{l}\text { No hay recomendaciones } \\
\text { de nivel I }\end{array}$ & $\begin{array}{l}\text { Mientras dure la ventila- } \\
\text { ción mecánica o la estan- } \\
\text { cia en } \mathrm{UCl}\end{array}$ & $\begin{array}{l}\text { Hasta ser capaz de tolerar la nutrición } \\
\text { enteral }\end{array}$ \\
\hline
\end{tabular}

lo tanto, se deben realizar aquellas que mejoren el uso de esta terapia en el paciente hospitalizado.

Fuerza de las recomendaciones, Clase I: ensayo clínico prospectivo y aleatorio; clase II: estudio prospectivo no comparativo o uno retrospectivo basado en datos fiables; clase III: serie de casos retrospectiva o revisión de bases de datos. ${ }^{48}$

\section{Referencias}

1. Marik P, Vasu T, Hirani A, Pachinburavan M. Stress ulcer prophylaxis in the new millenium: A systematic review and meta-analysis. Crit Care Med. 2010; 38:2222-28.

2. Grube RR, May DB. Stress ulcer prophylaxis in hospitalized patients not in intensive care units. Am J Health Syst Pharm. 2007 Jul 1; 64(13):1396-400.

3. Fennerty MB. Pathophysiology of the upper gastrointestinal tract in the critically ill patient: rationale for the therapeutic benefits of acid suppression. Crit Care Med. 2002 Jun; 30(6 Suppl):S351-5.

4. Mutlu GM, Mutlu EA, Factor P. GI complications in patients receiving mechanical ventilation. Chest. 2001;119(4):1222-41.

5. Metz DC. Preventing the gastrointestinal consequences of stress-related mucosa disease. Curr Med Res Opin. 2005 Jan; 21(1):11-8.

6. Quenot JP, Thiery N, Barbar S. When should stress ulcer prophylaxis be used in the ICU?. Curr Opin Crit Care. 2009; 15:139-43.

7. Amaral MC, Favas C, Alves JD, Riso N, Riscado MV. Stress-related mucosa disease: Incidence of bleeding and the role of omeprazole in its prophylaxis. Eur J Int Med. 2010; 21(5):386-88.
8. Qadeer MA, Richter JE, Brotman DJ. Hospital-acquired gastrointestinal bleeding outside the critical care unit: risk factors, role of acid suppression, and endoscopy findings. J Hosp Med. 2006 Jan; 1(1):13-20.

9. Herzig SJ, Vaughn BP, Howell MD, Ngo LH, Marcantonio ER. Acid-suppressive medication use and the risk for nosocomial gastrointestinal tract bleeding. Arch Intern Med. 2011 Jun 13; 171(11):991-7.

10. Cook DJ, Fuller HD, Guyatt GH, Marshall JC, Leasa D, Hall R, et al. Risk factors for gastrointestinal bleeding in critically ill patients. Canadian Critical Care Trials Group. N Engl J Med. 1994 Feb 10; 330(6):377-81.

11. Cook D, Heyland D, Griffith L, Cook R, Marshall J, Pagliarello J. Risk factors for clinically important upper gastrointestinal bleeding in patients requiring mechanical ventilation. Canadian Critical Care Trials Group. Crit Care Med. 1999 Dec; 27(12):2812-7.

12. Steinberg KP. Stress-related mucosal disease in the critically ill patient: risk factors and strategies to prevent stress-related bleeding in the intensive care unit. Crit Care Med. 2002 Jun; 30 (6 Suppl):S362-4.

13. Stollman N, Metz DC. Pathophysiology and prophylaxis of stress ulcer in intensive care unit patients. J Crit Care. 2005 Mar; 20(1):35-45.

14. Martindale RG. Contemporary strategies for the prevention of stress-related mucosal bleeding. Am J Health Syst Pharm. 2005 May 15; 62(10 Suppl 2):S11-7.

15. Terdiman JP, Ostroff JW. Gastrointestinal bleeding in the hospitalized patient: a case-control study to assess risk factors, causes, and outcome. Am J Med. 1998 Apr; 104(4):349-54

16. Estruch R, Pedrol E, Castells A, Masanes F, Marrades RM, Urbano-Marquez A. Prophylaxis of gastrointestinal tract bleeding with magaldrate in patients admitted to a general hospital ward. Scand J Gastroenterol. 1991 Aug; 26(8):819-26.

17. Grau JM, Casademont J, Fernandez-Sola J, Cardellach F, Urbano-Marquez A. Prophylaxis of gastrointestinal tract bleeding in patients admitted to a general hospital ward. Comparative study of sucralfate and cimetidine. Scand J Gastroenterol. 1993 Mar; 28(3):244-8.

18. ASHP Therapeutic Guidelines on Stress Ulcer Prophylaxis. ASHP Commission on Therapeutics and approved by the ASHP Board of Directors on November 14, 1998. Am J Health Syst Pharm. 1999 Feb 15; 56(4):347-79. 
19. Cook D, Guyatt G, Marshall J, Leasa D, Fuller H, Hall R, et al. A comparison of sucralfate and ranitidine for the prevention of upper gastrointestinal bleeding in patients requiring mechanical ventilation. N Engl J Med. 1998 Mar 19; 338(12):791-7.

20. Conrad SA, Gabrielli A, Margolis B, Quartin A, Hata JS, Frank WO, et al. Randomized, double blind comparison of immediate-release omeprazole-oral suspension versus intravenous cimetidina for prevention of upper gastrointestinal bleeding in critically ill patients. Crit Care Med. 2005; 33(4):760-65.

21. Cook DJ, Witt LG, Cook RJ, Guyatt GH. Stress ulcer prophylaxis in the critically ill. A meta-analysis. Am J Med. 1991 Nov; 91(5):519-27.

22. Tryba M. Prophylaxis of stress ulcer bleeding. A meta-analysis. J Clin Gastroenterol. 1991; 13 Suppl 2:S44-55.

23. Cook DJ, Reeve BK, Guyatt GH, Heyland DK, Griffith LE, Buckingham L, et al. Stress ulcer prophylaxis in critically ill patients. Resolving discordant metaanalyses. JAMA 1996; 275 (4): 308-14.

24. Lin $\mathrm{P}$, Chang $\mathrm{Ch}$, Hsu $\mathrm{P}$, Tseng $\mathrm{P}$, Huang $\mathrm{Y}$. The efficacy and safety of protom pump inhibitors vs histamine-2 receptor antagonist for stress ulcer bleeding prophylaxis among critical care patients: A metanalysis. Crit Care Med. 2010: 38(4): 1197-1205.

25. Pham CQ, Regal RE, Bostwick TR, Knauf KS. Acid suppressive therapy use on an inpatient internal medicine service. Ann Pharmacother. 2006 Jul-Aug; 40(78):1261-6.

26. Nardino RJ, Vender RJ, Herbert PN. Overuse of acid-suppressive therapy in hospitalized patients. Am J Gastroenterol. 2000 Nov; 95(11):3118-22.

27. Noguerado Asensio A, Rodriguez Barrientos R, Zelaya Castro P, Sanchez Sempere A, Antuna Blanco F, Lutz Garcia E, et al. [Use of acid-suppressive medications in hospitalized patients]. An Med Interna. 2002 Nov; 19(11):557-60.

28. Zink DA, Pohlman M, Barnes M, Cannon ME. Long-term use of acid suppression started inappropriately during hospitalization. Aliment Pharmacol Ther 2005 May 15; 21(10):1203-9.

29. Khudair IF, Sadik ND, Hanssens YI. Prescribing pattern of acid suppressive medications for medical inpatients in a teaching hospital in Qatar. Saudi Med J. 2009 Jan; 30(1):125-9.

30. Zeitoun A, Zeineddine M, Dimassi H. Stress ulcer prophylaxis guidelines: Are they being implemented in Lebanese health care centers? World J Gastrointest Pharmacol Ther. 2011; 2(4): 27-35

31. Bez C, Perrottet N, Zingg T, Leung Ki EL, Demartines N, Pannatier A. Stress ulcer prophylaxis in non-critically ill patients: a prospective evaluation of current practice in a general surgery department. J Eval Clin Pract. 2013 Apr; 19(2):374-8

32. Gupta R, Garg P, Kottoor R, Munoz JC, Jamal MM, Lambiase LR, et al. Overuse of Acid Suppression Therapy in Hospitalized Patients. South Med J. 2010; 103(3): 207-11.

33. Hussain S, Stefan M, Visintainer P, Rothberg M. Why Do Physicians Prescribe Stress Ulcer Prophylaxis to General Medicine Patients? Sout Med J. 2010; 103(11): 1103-10.

34. Issa I, Soubra O, Nakkash H, Soubra L. Variables Associated with Stress Ulcer Prophylaxis Misuse: A Retrospective Analysis. Dig Dis Sci. 2012 Oct; 57(10):2633-41.

35. Dial S, Delaney JA, Barkun AN, Suissa S. Use of gastric acid-suppressive agents and the risk of community-acquired Clostridium difficile-associated disease. JAMA. 2005 Dec 21; 294(23):2989-95.
36. Dial S, Alrasadi K, Manoukian C, Huang A, Menzies D. Risk of Clostridium difficile diarrhea among hospital inpatients prescribed proton pump inhibitors: cohort and case-control studies. CMAJ. 2004 Jul 6; 171(1):33-8.

37. Lowe DO, Mamdani MM, Kopp A, Low DE, Juurlink DN. Proton pump inhibitors and hospitalization for Clostridium difficile-associated disease: a population-based study. Clin Infect Dis. 2006 Nov 15; 43(10):1272-6.

38. Laheij RJ, Sturkenboom MC, Hassing RJ, Dieleman J, Stricker BH, Jansen JB. Risk of community-acquired pneumonia and use of gastric acid-suppressive drugs. JAMA. 2004 Oct 27; 292(16):1955-60.

39. Herzig SJ, Howell MD, Ngo LH, Marcantonio ER. Acid-suppressive medication use and the risk for hospital-acquired pneumonia. JAMA. 2009 May 27; 301(20):2120-8.

40. Laine L. Protom pump inhibitors and bone fractrures?. 2009 Mar; 104 Suppl 2:S21-6.

41. Eom CS, Park SM, Myung SK, Yun JM, Ahn JS. Use of Acid- Suppressive Drugs and Risk of Fracture: A Meta-analysis of Observational Studies. Ann Fam Med. 2011; 11(9): 257-67.

42. Erstad BL, Camamo JM, Miller MJ, Webber AM, Fortune J. Impacting cost and appropriateness of stress ulcer prophylaxis at a university medical center. Crit Care Med. 1997 Oct; 25(10):1678-84.

43. Wadibia EC, Lucas BD, Hilleman DE, Mooss AN. Economic impact of inappropiate histamine 2-receptor antagonist use. Clin Ther. 1997 Sep-Oct; 19(5):1085-91.

44. Heidelbaugh JJ, Inadomi JM. Magnitude and economic impact of inappropriate use of stress ulcer prophylaxis in non-ICU hospitalized patients. Am J Gastroenterol. 2006 Oct; 101(10):2200-5.

45. Saultz A, Judkins DZ, Saultz JW, Fashner J. What Gl stress ulcer prophylaxis should we provide hospitalized patients? J Fam Prac. 2007; 56(1): 51-2.

46. Kleb FH, Scholmerich J. Future expectations in the prophylaxis of intestinal bleeding. Best Pract Res Clin Gastroenterol. 2008; 22(2):373-87.

47. Lanza F, Chan F, Quigley E. Guidelines for prevention of NSAID-Related ulcer complications. Am J Gastroenterol. 2009; 104(3): 728-38.

48. Guillamondegui OD, Gunter OL Jr, Bonadies JA, Coates JE, Kurek SJ, De Moya MA. Practice management guidelines for stress ulcer prophylaxis [monografía en Internet]. Rockville, MD: Agency for Healthcare Research and Quality; 2008 [citado 24 Jul 2013]. Disponible en: http://guidelines.gov/content. aspx?id=12635.

49. Dworzynski K, Pollit V, Kelsey A, Higgins B, Palmer K. Management of acute upper gastrointestinal bleeding: summary of NICE guidance. BMJ. 2012 Jun 13; 344:e3412.

50. Yachimski PS, Farrell EA, Hunt DP, Reid AE. Proton pump inhibitors for prophylaxis of nosocomial upper gastrointestinal tract bleeding: effect of standardized guidelines on prescribing practice. Arch Intern Med. 2010 May 10; 170(9):779-83.

51. Khalili H, Dashti-Khavidaki S, Hossein Talasaz A, Tabeefar H, Hendoiee N. Descriptive Analysis of a Clinical Pharmacy Intervention to Improve the Appropriate Use of Stress Ulcer Prophylaxis in a Hospital Infectious Disease Ward. J Manag Care Pharm. 2010; 16(2):114-21

52. Coursol C, Sanzari S. Impact of Stress Ulcer Prophylaxis Algorithm Study. Ann Pharmacother. 2005; 39:810-6. 